\title{
Rotor-Router Aggregation on the Comb
}

\author{
Wilfried Huss \\ Department of Mathematics \\ Siegen University \\ Walter-Flex-Strasse 3 \\ 57068 Siegen, Germany \\ huss@mathematik. uni-siegen.de
}

\author{
Ecaterina Sava \\ Department of Mathematics C \\ Graz University of Technology \\ Steyrergasse 30/III \\ 8010 Graz, Austria \\ sava@tugraz.at
}

Submitted: Jun 05, 2011; Accepted: Nov 10, 2011; Published: Nov 21, 2011

Mathematics Subject Classification: 82C20, 60J10, 05C81

\begin{abstract}
We prove a shape theorem for rotor-router aggregation on the comb, for a specific initial rotor configuration and clockwise rotor sequence for all vertices. Furthermore, as an application of rotor-router walks, we describe the harmonic measure of the rotor-router aggregate and related shapes, which is useful in the study of other growth models on the comb. We also identify the shape for which the harmonic measure is uniform. This gives the first known example where the rotor-router cluster has non-uniform harmonic measure, and grows with different speeds in different directions.
\end{abstract}

Keywords: growth model, comb, rotor-router, asymptotic shape, harmonic measure.

\section{Introduction}

Rotor-router walks are deterministic analogues to random walks, which have been introduced into the physics literature under the name Eulerian walks by PRIEZzHEV, D.DHAR ET AL [PDDK96] as a model of self organized criticality, a concept established by BAK, TANG AND WIESENFELD [BTW88].

In a rotor-router walk on a graph $G$, for each vertex $x \in G$ a cyclic ordering $c(x)$ of its neighbours is chosen. At each vertex we have an arrow (rotor) pointing to one of the neighbours of the vertex. A particle performing a rotor-router walk carries out the following procedure at each step: first it changes the rotor at its current position $x$ to point to the next neighbour of $x$ defined by the ordering $c(x)$, and then the particle moves to the neighbour the rotor is now pointing at. 
The behaviour of rotor-router walks is in some respects remarkably close to that of random walks. See for example CoOper And Spencer [CS06] and DoERr And Friedrich DF06].

In the present paper, we are interested in a process called rotor-router aggregation, defined as follows. Choose a root vertex $o \in G$ and let $R_{1}=\{o\}$. The sets $R_{n}$ are defined recursively, by

$$
R_{n+1}=R_{n} \cup\left\{z_{n}\right\} \quad \text { for } n \geq 1,
$$

where $z_{n}$ is the first vertex outside $R_{n}$ that is visited by a rotor-router walk started at the origin $o$. The rotor configuration is not changed when a new particle is started at the origin. We will call the set $R_{n}$ the rotor-router cluster of $n$ particles.

Rotor-router aggregation on the Euclidean lattice $\mathbb{Z}^{d}$ has been studied by LEVINE AND PERES [LP09], who showed that the rotor-router cluster $R_{n}$ forms a ball in the usual Euclidean distance. On the homogeneous tree LANDAU AND LEVINE [LL09] proved that, under certain conditions on the initial configuration of rotors, the rotor-router cluster $R_{n}$ forms a perfect ball with respect to the graph metric, whenever it has the right amount of particles. KAGER AND LEVINE [KL10] studied the shape of the rotor-router cluster on a modified two-dimensional lattice, which they call the layered square lattice.

In each of these examples the fluctuations of the cluster around the limiting shape are much smaller in rotor-router aggregation than in the corresponding random growth model called internal diffusion limited aggregation (IDLA), where particles perform independent random walks before they settle and attach to the cluster. In the case of the homogeneous tree and the layered square lattice, the fluctuations even vanish completely in the deterministic model.

We will use the technique introduced in [KL10] in order to study rotor-router aggregation on the two-dimensional comb $\mathcal{C}_{2}$, which is the spanning tree of the two-dimensional lattice $\mathbb{Z}^{2}$, obtained by removing all horizontal edges of $\mathbb{Z}^{2}$ except the ones on the $x$-axis. In other words, the graph $\mathcal{C}_{2}$ can be constructed from a two-sided infinite path $\mathbb{Z}$ (the "backbone" of the comb), by attaching copies of $\mathbb{Z}$ (the "teeth") at every vertex of the backbone.

We use the standard embedding of the comb into the two-dimensional Euclidean lattice $\mathbb{Z}^{2}$, and use Cartesian coordinates $z=(x, y) \in \mathbb{Z}^{2}$ to denote vertices of $\mathcal{C}_{2}$. The vertex $o=(0,0)$ will be the root vertex, see Figure $1(\mathrm{a})$. For functions $g$ on the vertex set of $\mathcal{C}_{2}$ we will often write $g(x, y)$ instead of $g(z)$, when $z=(x, y)$.

While $\mathcal{C}_{2}$ is a very simple graph, it has some remarkable properties. For example, the Einstein relation between the spectral-, walk- and fractal-dimension is violated on the comb, see Bertacchi [Ber06]. Peres and Krishnapur [KP04 showed that on $\mathcal{C}_{2}$ two independent simple random walks meet only finitely often; this is the so-called finite collision property.

The structure of this paper is as follows. In Section 2 we recall some preliminary results due to KAGER AND LEVINE [KL10, which will be applied in order to prove the main result of the paper. In Section 3 we describe the shape of the rotor-router cluster 


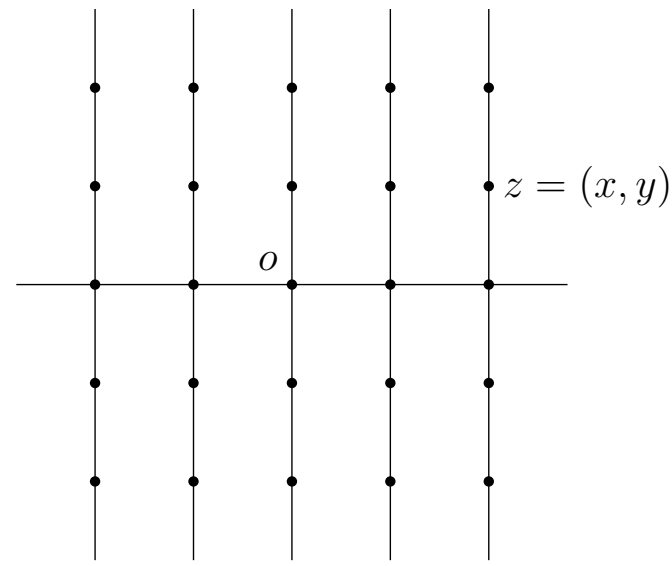

(a)

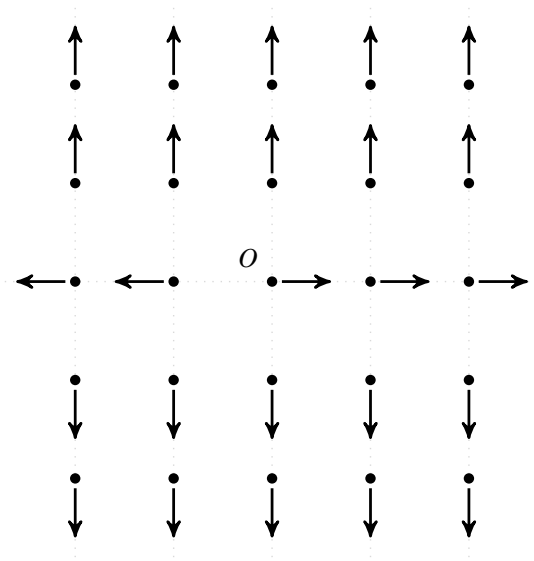

(b)

Figure 1: (a) The two-dimensional comb $\mathcal{C}_{2}$. (b) The initial rotor configuration $\rho_{0}$.

on $\mathcal{C}_{2}$, for the initial rotor configuration $\rho_{0}$ in Figure $1(\mathrm{~b})$. Define

$$
B_{m}=\left\{(x, y) \in \mathcal{C}_{2}:|x| \leq m,|y| \leq h(m-|x|)\right\} \quad \text { for } m \in \mathbb{N},
$$

for some function $h: \mathbb{N}_{0} \rightarrow \mathbb{N}_{0}$. The main result of the paper is the following.

Theorem 1.1. Let $R_{n}$ be the rotor-router cluster of $n$ particles on the comb $\mathcal{C}_{2}$, with initial rotor configuration as in Figure $1(b)$ and clockwise rotor sequence for all $x \in \mathcal{C}_{2}$. Let $B_{m}$ be as in (1) with

$$
h(x)=\left\lfloor\frac{(x+1)^{2}}{3}\right\rfloor .
$$

Then, for all $m \geq 0$ and $n_{m}=\left|B_{m}\right|$, the rotor-router cluster $R_{n_{m}}$ satisfies $R_{n_{m}}=B_{m}$.

The main idea of the proof is to study rotor-router aggregation on the non-negative integers $\mathbb{N}_{0}$, and then to glue different copies of $\mathbb{N}_{0}$ on the "backbone" of $\mathcal{C}_{2}$, in order to get information on the behaviour of the rotor-router cluster. In the upcoming paper [HS11] the authors study IDLA on the comb. This gives another case where all fluctuations disappear in rotor-router aggregation when compared with IDLA.

As an application of rotor-router walks, in Section 4 we give a method to describe the harmonic measure of generic sets $B_{m}$, of the form (1). For this, let

$$
\partial B_{m}=\left\{z \in B_{m}: \exists \text { neighbour } y \text { of } z \text { in } \mathcal{C}_{2} \text {, such that } y \notin B_{m}\right\}
$$

be the inner boundary of the set $B_{m}$. The harmonic measure of $B_{m}$ is defined as the exit distribution of a simple random walk from the set $B_{m}$. For $z \in \partial B_{m}$, we denote by $\nu_{m, o}(z)$ the probability that a simple random walk started at the origin $o \in \mathcal{C}_{2}$ hits $\partial B_{m}$ in $z$. In order to compute $\nu_{m, o}(z)$, we consider a special rotor-router process, which allows us to obtain exact results in several cases. In particular, we identify the subsets of the comb for which the harmonic measure is uniform. 
Theorem 1.2. Let $B_{m} \subset \mathcal{C}_{2}$ be as in (1), with $h(x)=x^{2}$. Then the harmonic measure $\nu_{m, o}$ of $B_{m}$ is the uniform measure on $\partial B_{m}$.

For the router-router cluster in Theorem 1.1, we are able to give asymptotics of the harmonic measure. We prove the following.

Theorem 1.3. Let $B_{m} \subset \mathcal{C}_{2}$ be as in (1) with $h(x)=\left\lfloor(x+1)^{2} / 3\right\rfloor$, and $z=\left(z_{x}, z_{y}\right) \in$ $\partial B_{m}$. There exists a function $e: \mathbb{N}_{0} \rightarrow \mathbb{N}$ with $\lim _{x \rightarrow \infty} \frac{e(x)}{x}=c$, and $0<c<1 / 2$, such that for all $m \geq 0$ the harmonic measure $\nu_{m, o}(z)$ is proportional to e $\left(m-\left|z_{x}\right|\right)$.

This gives the first example where the rotor-router cluster is not a set with uniform harmonic measure, and grows faster in the vertical direction than in the horizontal direction.

We want to emphasize that it is not easy to apply this method in many cases, since it requires exact knowledge of the odometer function of the rotor-router walk, and at least some insight in the structure of the Abelian sandpile group of the set under consideration. The connection of the Abelian sandpile group to the rotor-router model has been established in the physics literature; see [PPS98, PDDK96]. One can define a group based on the action of a particle which performs a rotor-router walk on the rotor configuration. This rotor-router group is abelian and isomorphic to the Abelian sandpile group. This isomorphism has been proven formally in [LL09]. For a self-contained introduction see the overview paper of Holroyd, Levine, ET.AL. [HLM+08].

\section{Preliminaries}

Let $(G, E(G))$ be an infinite, undirected and connected graph, with vertex set $G$, equipped with a symmetric adjacency relation $\sim$, which defines the set of edges $E(G)$ (as a subset of $G \times G)$. We write $(x, y)$ for the edge between the pair of neighbours $x, y$. In order to simplify the notation, instead of writing $(G, E(G))$ for a graph, we shall write only $G$, and it will be clear from the context whether we are considering edges or vertices. We denote by $d(x)$ the degree of the vertex $x$, that is, the number of neighbours of $x$ in $G$. Fix a nonempty subset $S \subset G$ of vertices called sinks, and let $G^{\prime}=G \backslash S$.

The odometer function $u(x)$ of the rotor-router aggregation is defined as the number of particles sent out by the vertex $x$ during the creation of the rotor-router cluster $R_{n}$ of $n$ particles.

A rotor configuration on $G$ is a function $\rho: G^{\prime} \rightarrow G$, such that $\rho(x)$ is a neighbour of $x$, for all $x \in G^{\prime}$, that is $(x, \rho(x)) \in E(G)$. Hence, $\rho$ assigns to every vertex one of its neighbours. A rotor configuration $\rho$ is called acyclic, if the subgraph of $G$ spanned by the rotors contains no directed cycles. A particle configuration on $G$ is a function $\sigma: G \rightarrow \mathbb{Z}$, with finite support. If $\sigma(x)=m>0$, we say that there are $m$ particles at vertex $x$. The rotor sequence at vertex $x$ will be denoted by $c(x)=\left(x_{0}, x_{1}, \ldots, x_{d(x)-1}\right)$ where all $x_{i} \sim x$ and $x_{i} \neq x_{j}$ for $i \neq j$, with $i, j=0,1, \ldots, d(x)-1$. If $y=x_{i} \in c(x)$, for some $i \in\{0,1, \ldots, d(x)-1\}$, we denote by $y^{+}$the vertex $x_{(i+1) \bmod } d(x)$. 
Definition 2.1 (Toppling operator). Fix a vertex $x \in G^{\prime}$. For a rotor configuration $\rho$ and a particle configuration $\sigma$ on $G$, we define the toppling operator $F_{x}$, which sends one particle out of vertex $x$, by

$$
F_{x}(\rho, \sigma)=\left(\rho^{\prime}, \sigma^{\prime}\right)
$$

where the new rotor configuration $\rho^{\prime}$ is given by

$$
\rho^{\prime}(y)= \begin{cases}\rho(y)^{+} & \text {if } y=x, \\ \rho(y) & \text { otherwise },\end{cases}
$$

and the new particle configuration $\sigma^{\prime}$ is given by

$$
\sigma^{\prime}(y)= \begin{cases}\sigma(y)-1 & \text { if } y=x, \\ \sigma(y)+1 & \text { if } y=\rho^{\prime}(x), \\ \sigma(y) & \text { otherwise. }\end{cases}
$$

So $F_{x}$ first changes the rotor configuration by rotating the arrow at $x$ to its next position in the cyclic ordering $c(x)$, and then it sends a particle to the vertex the rotor at $x$ is now pointing at. The operation $F_{x}$ of toppling at some vertex $x$ can be successful even if there is no particle at $x$. If this is the case, then a "virtual particle" is sent away from $x$ and a "hole" is left there. If there is already a hole at $x$, the operator $F_{x}$ will increase its depth by one. In the normal rotor-router aggregation no holes are ever created during the whole process. A sequence of topplings $\left\{x_{k}\right\}_{k \geq 1}$ is called legal, if no holes are created when the vertices $x_{k}$ are toppled in sequence.

Note that the toppling operators commute, i.e., $F_{x} F_{y}=F_{y} F_{x}$ for all $x, y \in G^{\prime}$. This is the usual abelian property for rotor-router walks. While the final configuration is always the same, rearranging the order of the topplings can turn a legal toppling sequence into one that creates holes and virtual particles.

Given a function $u: G^{\prime} \rightarrow \mathbb{N}$, let

$$
F^{u}=\prod_{x \in G^{\prime}} F_{x}^{u(x)},
$$

where product means composition of the operators. Because of the abelian property, $F^{u}$ is well defined.

In order to prove a shape result for rotor-router aggregation on $\mathcal{C}_{2}$, for a specific initial configuration, we will apply a stronger version of the usual Abelian property of rotorrouter walks, which has been recently introduced by KAGER AND LEvine [KL10]. We state it here for completeness.

Theorem 2.2 (Strong Abelian Property). Let $\rho_{0}$ be a rotor configuration and $\sigma_{0}$ a particle configuration on $G$. Given two functions $u_{1}, u_{2}: G^{\prime} \rightarrow \mathbb{N}$, write

$$
F^{u_{i}}\left(\rho_{0}, \sigma_{0}\right)=\left(\rho_{i}, \sigma_{i}\right), \quad i=1,2 .
$$

If $\sigma_{1}=\sigma_{2}$ on $G^{\prime}$, and both $\rho_{1}$ and $\rho_{2}$ are acyclic, then $u_{1}=u_{2}$. 
Note that the equality $u_{1}=u_{2}$ implies also that $\rho_{1}=\rho_{2}$, and moreover $\sigma_{1}=\sigma_{2}$ on all of $G$. This result allows us to drop the hypothesis of legality: each final particle configuration can only be achieved by an unique amount of topplings for each vertex, even if we allow virtual particles to be formed during the process.

Friedrich And Levine [FL11] used the Strong Abelian Property to give an exact characterization of the odometer function of rotor-router aggregation. Recall that the odometer function $u(x)$ at some vertex $x$ represents the number of particles sent out by $x$ during the creation of the rotor-router cluster.

Theorem 2.3 (Friedrich, Levine). Let $G$ be a finite or infinite directed graph, $\rho_{0}$ an initial rotor configuration on $G$, and $\sigma_{0}=n \cdot \delta_{o}$. Fix $u_{\star}: G \rightarrow \mathbb{N}$, and let

$$
A_{\star}=\left\{x \in G: u_{\star}(x)>0\right\} .
$$

Further define $\rho_{\star}$ and $\sigma_{\star}$ by

$$
F^{u_{\star}}\left(\rho_{0}, \sigma_{0}\right)=\left(\rho_{\star}, \sigma_{\star}\right) .
$$

Suppose the following properties hold

(a) $\sigma_{\star} \leq 1$,

(b) $A_{\star}$ is finite,

(c) $\sigma_{\star}(x)=1$ for all $x \in A_{\star}$, and

(d) $\rho_{\star}$ is acyclic on $A_{\star}$.

Then $u_{\star}$ is the rotor-router odometer function of $n$ particles.

Using Theorem 2.3, for the proof of Theorem 1.1 it is enough to give an explicit formula for the corresponding odometer function, and to check if it satisfies all the required properties.

\section{Rotor-Router Aggregation}

Consider now the rotor-router aggregation on the comb $\mathcal{C}_{2}$, and the initial rotor configuration as in Figure 1(b), Through this section, $B_{m}$ will be the set defined in (1), with $h(x)$ given by

$$
h(x)=\left\lfloor\frac{(x+1)^{2}}{3}\right\rfloor .
$$

Definition 3.1. Let $(\rho, \sigma)$ be the final configuration of the rotor-router aggregation process of $\left|B_{m}\right|$ particles described in Theorem 1.1. The configuration $(\rho, \sigma)$ is then called the $m$-th fully symmetric configuration. 


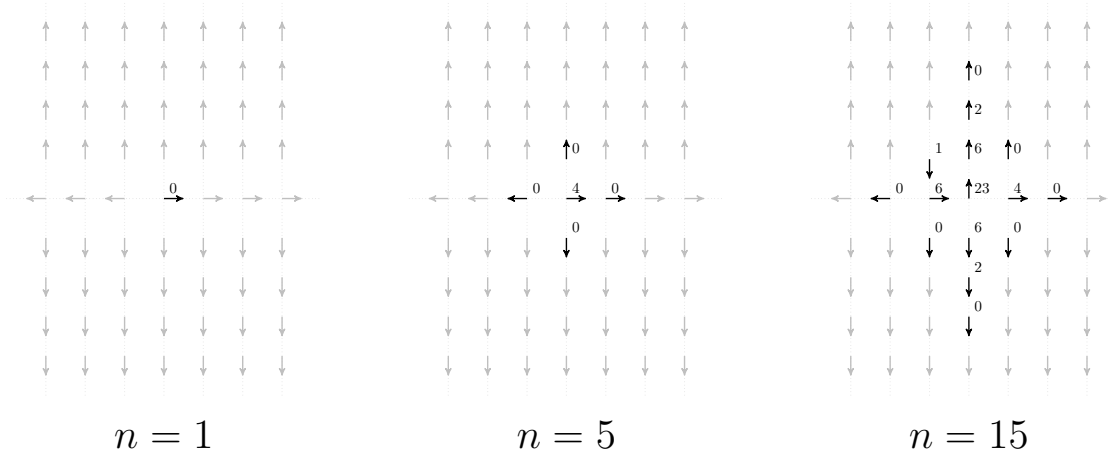

Figure 2: The first three fully symmetric configurations, consisting of $n$ particles. The numbers on the arrows are the values of the odometer function $u_{n}$.

Figures 2 and 3 show examples of fully symmetric configurations. In Figure 3 , one can observe that the fully symmetric configuration of $\left|B_{7}\right|$ particles, as well as its corresponding rotor-router odometer function, are obtained by shifting "half" of the configuration of $\left|B_{6}\right|$ particles one step in the direction of the positive resp. negative $x$-axis and filling in the values for the "tooth" corresponding to $x=0$. It turns out that this is true for all fully symmetric configurations (with the exception of the first 3 ). This property will play an important role in the proof of Theorem 1.1 .

For proving Theorem 1.1, an exact expression for the cardinality of the sets $B_{m}$ is needed.

Lemma 3.2. Let $B_{m}$ be the set defined in (1), with $h(x)=\left\lfloor\frac{(x+1)^{2}}{3}\right\rfloor$. Then, for all $m \geq 0$ the cardinality of $B_{m}$ is given by

$$
\left|B_{m}\right|=\frac{1}{9}\left[4 m^{3}+12 m^{2}+24 m+5+2((m+2) \bmod 3)\right] .
$$

Proof. In order to simplify the statement of the Lemma, we have to distinguish three cases, namely for $m=3 k+i$, with $i=0,1,2$. The right-hand side of (3) is then equal to

$$
\begin{aligned}
& N_{0}(k)=12 k^{3}+12 k^{2}+8 k+1, \text { for } m=3 k \\
& N_{1}(k)=12 k^{3}+24 k^{2}+20 k+5, \text { for } m=3 k+1 \\
& N_{2}(k)=12 k^{3}+36 k^{2}+40 k+15, \text { for } m=3 k+2 .
\end{aligned}
$$

Moreover, in the three cases $m=3 k+i$, with $i=0,1,2$, the function $h$ can be written as follows.

$$
\begin{aligned}
h(3 k) & =3 k^{2}+2 k \\
h(3 k+1) & =3 k^{2}+4 k+1 \\
h(3 k+2) & =3 k^{2}+6 k+3 .
\end{aligned}
$$

We prove (4) by induction on $k$. The base case $m=1$ is immediate from the definition of $B_{m}$. With

$$
\left|B_{m+1}\right|=\left|B_{m}\right|+2[h(m)+h(m+1)+1]
$$


follows the inductive step.

Next, we will find an exact formula for the odometer function of the rotor-router aggregation defined in Theorem 1.1, and we shall prove its correctness using Theorem 2.3. For this, we first have a detailed look at the rotor-router process on the non-negative integers.

\subsection{Rotor-Router on the non-negative Integers}

For a better understanding of the rotor-router process on the comb $\mathcal{C}_{2}$, we first analyse it on the half-line, where it is very simple. Consider $G=\mathbb{N}_{0}$, with sink vertex 0 , and the initial rotor configuration $\tilde{\rho}_{0}: \mathbb{N} \rightarrow \mathbb{N}_{0}$ given by

$$
\tilde{\rho}_{0}(y)=y+1, \quad \text { for all } y \in \mathbb{N} .
$$

Let $\tilde{R}_{1}=\{1\}$, and define a modified rotor-router aggregation process $\tilde{R}_{n}$ recursively as follows. Start a rotor-router walk in 1, and stop the particle when it either reaches the sink 0 , or exits the previous cluster $\tilde{R}_{n-1}$. Denote by $\tilde{z}_{n}$ the vertex where the $n$-th particle stops, and by $\tilde{\rho}_{n}$ and $\tilde{u}_{n}$ the rotor configuration and odometer function at that time. Then,

$$
\tilde{R}_{n}= \begin{cases}\tilde{R}_{n-1} \cup\left\{\tilde{z}_{n}\right\}, & \text { if } \tilde{z}_{n} \neq 0 \\ \tilde{R}_{n-1}, & \text { otherwise. }\end{cases}
$$

Obviously $\tilde{R}_{n}=\{1, \ldots, \tilde{h}(n)\}$ for some sequence $\tilde{h}(n)$. Since $\tilde{\rho}_{0}$ is acyclic, all rotor configurations $\tilde{\rho}_{n}$ are acyclic and have the form

$$
\tilde{\rho}_{n}(y)= \begin{cases}y-1, & 0 \leq y \leq \tilde{r}(n) \\ y+1, & \text { otherwise }\end{cases}
$$

for some numbers $0 \leq \tilde{r}(n) \leq \tilde{h}(n)$. Here, $\tilde{r}(n)$ represents the vertex where the rotors change direction: all rotors up to $\tilde{r}(n)$ point inwards $(\downarrow)$, and all rotors from $\tilde{r}(n)+1$ up to $\tilde{h}(n)$ point outwards $(\uparrow)$.

For numbers $h, r$ and $y$ in $\mathbb{N}_{0}$, with $0 \leq r \leq h$ define the function $\tilde{u}$ as

$$
\tilde{u}(h, r, y)= \begin{cases}f(h-y)+e(r-y), & 1 \leq y \leq r \\ f(h-y), & r<y \leq h \\ 0, & \text { otherwise }\end{cases}
$$

where the functions $e$ and $f$ are given by $e(y)=2 y+1$ and $f(y)=y(y+1)$.

The odometer function $\tilde{u}(n)$ of the rotor-router process $\tilde{R}_{n}$ can now be defined in terms of (6) by setting

$$
\tilde{h}(n)=\max \left\{k \in \mathbb{N}: \frac{k(k+1)}{2} \leq n\right\}, \quad \tilde{r}(n)=n-\frac{\tilde{h}(n)(\tilde{h}(n)+1)}{2}
$$



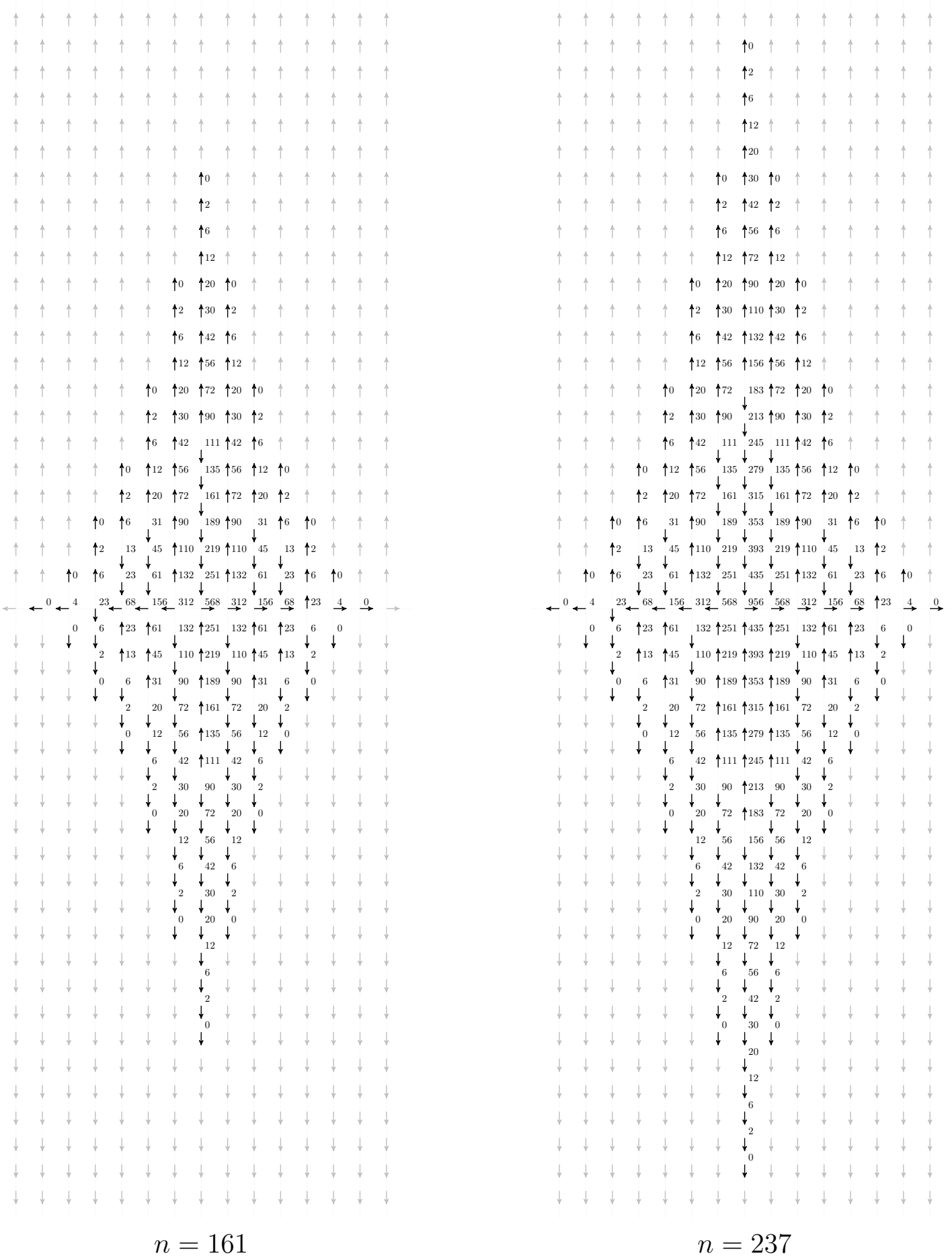

Figure 3: The $6^{\text {th }}$ and $7^{\text {th }}$ fully symmetric configurations, consisting of $\left|B_{6}\right|$ and $\left|B_{7}\right|$ particles. The numbers are the values of the odometer function $u_{6}$ and $u_{7}$, respectively. 


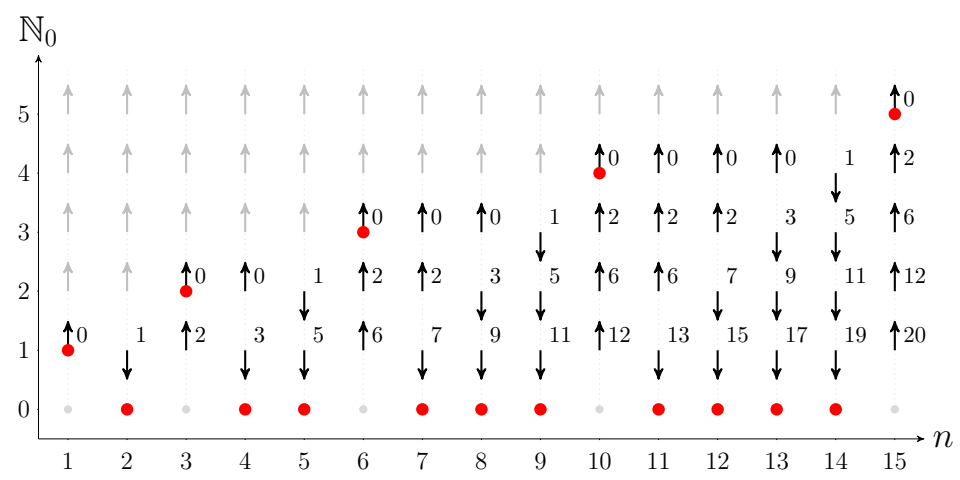

Figure 4: The first steps of the process $\tilde{R}_{n}$ on $\mathbb{N}$. The dots mark the vertex where the current particle stopped.

and

$$
\tilde{u}_{n}(y)=\tilde{u}(\tilde{h}(n), \tilde{r}(n), y),
$$

for $\tilde{h}(n)$ and $\tilde{r}(n)$ as defined in (7). It is easy to verify by induction that $\tilde{u}_{n}$ correctly describes the odometer function of $\tilde{R}_{n}$. See Figure 4 for a graphical representation of the process $\tilde{R}_{n}$.

\subsection{Rotor-Router on the Comb}

Since the rotor-router aggregation on the "half-teeth" of $\mathcal{C}_{2}$ behaves like the process $\tilde{R}_{n}$ from the previous section, it is enough to determine the numbers $h$ and $r$ in (6), which now depend on $x$ and the number of particles, in order to fully specify the odometer function on $\mathcal{C}_{2}$ for points off the $x$-axis.

Let $B_{m}$ as defined in (1), with $h(x)=\left\lfloor\frac{(x+1)^{2}}{3}\right\rfloor$ and define $r(x)$ by

$$
r(x)= \begin{cases}0, & x \in\{0,1\} \\ \frac{1}{18}\left(x^{2}-7 x+10\right), & x \equiv 2 \bmod 3 \\ \frac{1}{6}\left(x^{2}-x+6\right), & \text { otherwise. }\end{cases}
$$

Define $u_{m}: B_{m} \rightarrow \mathbb{N}$ by

$$
u_{m}(x, y)=u^{\prime}(m-|x|,|y|)
$$

where

$$
u^{\prime}(x, y)= \begin{cases}\tilde{u}(h(x), r(x), y), & y>0 \\ 2 f(h(x))+2 e(r(x))-2-\mathbb{1}_{\{x=2\}}, & y=0\end{cases}
$$

with $\tilde{u}$ as in (6), $h(x)$ and $r(x)$ defined as above, and $e(x)=2 x+1$ and $f(x)=x(x+1)$. We claim that $u_{m}$ is the odometer function for rotor-router aggregation of $\left|B_{m}\right|$ particles on the comb. 


\subsubsection{Proof of Theorem 1.1}

Let $B_{m}$ as defined in (1), with $h(x)=\left\lfloor(x+1)^{2} / 3\right\rfloor$ as in Theorem 1.1, and $u_{m}$ defined as in 10$)$.

Lemma 3.3. Let $\rho_{0}$ be the initial rotor configuration defined in Figure 1(b), and the initial particle configuration $\sigma_{0}=\left|B_{m}\right| \cdot \delta_{o}$. Furthermore, define $\rho_{m}$ and $\sigma_{m}$ as

$$
\left(\rho_{m}, \sigma_{m}\right)=F^{u_{m}}\left(\rho_{0}, \sigma_{0}\right)
$$

with $u_{m}$ as in (10). If a clockwise rotor sequence is assumed for all vertices, then $u_{m}$ is the odometer function of the rotor-router aggregation with $\left|B_{m}\right|$ particles and moreover $\sigma_{m}=\mathbb{1}_{B_{m}}$, for all $m \geq 3$.

Proof. To verify that $u_{m}$ is indeed the odometer function of this rotor-router process, we need to check the four properties of Theorem 2.3, with

$$
A_{\star}=B_{m} \backslash \partial B_{m}
$$

where $\partial B_{m}$ is the inner boundary of $B_{m}$ defined in (2). The set $A_{\star}$ is obviously finite. For those vertices $z \in A_{\star}$ that have neighbours in $B_{m} \backslash A_{\star}$, we have by (8) and (6) that $u_{m}(z) \leq 3$ if $z$ is not on the $x$-axis, and $u_{m}(z)=4$ otherwise. In both cases at most one particle is sent to some vertex outside of $A_{\star}$, hence $\sigma_{m}(z) \leq 1$ for all $z \notin A_{\star}$.

Next we verify that the final particle configuration $\sigma_{m}$ is equal to 1 on $A_{\star}$. Since by definition $u_{m}$ is symmetric, it is enough to consider only one quadrant. Additionally, we shift the coordinate system such that the point $(-m, 0)$ lies at the origin, which means that we can work with the function $u^{\prime}$, defined in (11). Since $u^{\prime}$ does not depend on the parameter $m$, most of what follows holds independently of $m$. Only for the center $(m, 0)$ of the set $B_{m}$ (Case 4$)$, we need to take the parameter $m$ into account. Let $z=(x, y) \in A_{\star}$ with $x, y \geq 0$. We distinguish several cases.

Case 1. $y \geq 2$ : For vertices $(x, y)$, with $y \geq 2$, the rotor-router aggregation behaves as the process $\tilde{R}_{n}$, defined in Section 3.1. From Figure 4, and due to the fact that the final rotor configuration restricted to each "tooth" is acyclic, there are again four possible situations:

(a) The rotors at the vertices $(x, y-1),(x, y)$ and $(x, y+1)$ all point outwards $(\uparrow)$. This is the case when $r(x)<y-1$, hence the vertex $(x, y)$ receives $\frac{1}{2} \tilde{u}_{m}(y-1)+\frac{1}{2} \tilde{u}_{m}(y+1)$ particles from its upper and lower neighbours, and it sends $\tilde{u}_{m}(y)$ particles. That is,

$$
\sigma_{m}(x, y)=\frac{1}{2}[f(h(x)-y+1)+f(h(x)-y-1)]-f(h(x)-y)=1 .
$$

(b) The rotors at the vertices $(x, y-1),(x, y)$ and $(x, y+1)$ all point inwards $(\downarrow)$. Hence $r(x) \geq y+1$ and, comparing the numbers of incoming and outgoing particles, we have

$$
\begin{aligned}
\sigma_{m}(x, y)= & \frac{1}{2}[f(h(x)-y+1)+e(r(x)-y+1)+f(h(x)-y-1) \\
& +e(r(x)-y-1)]-f(h(x)-y)-e(r(x)-y)=1 .
\end{aligned}
$$


(c) When the rotors from 1 to $y-1$ point inwards $(\downarrow)$ and from $y$ to $h(x)$ point outwards $(\uparrow)$, then $r(x)=y-1$, and we have

$$
\begin{aligned}
\sigma_{m}(x, y)= & \frac{1}{2}[f(h(x), r(x), y-1)+e(0)-1] \\
& +\frac{1}{2} f(h(x), r(x), y+1)-f(h(x), r(x), y)=1
\end{aligned}
$$

(d) The last case which can appear is when all rotors from $y$ to 0 point inwards $(\downarrow)$, and from $y+1$ to $h(x)$ outwards $(\uparrow)$. Then $r(x)=y$ and

$$
\begin{aligned}
\sigma_{m}(x, y)= & \frac{1}{2}[f(h(x), r(x), y-1)+e(1)-1] \\
& +\frac{1}{2} f(h(x), r(x), y+1)-f(h(x), r(x), y)-1=1
\end{aligned}
$$

Therefore $\sigma(x, y)=1$ for all $(x, y) \in B_{m}$ with $y \geq 2$. Moreover, no closed cycle is formed by these vertices in the final rotor configuration $\rho_{m}$.

Case 2. $y=1$ : Consider $\sigma_{m}(z)$ for the vertex $z=(x, 1)$. For $x \geq 9$ the number of inwards pointing rotors $r(x)$ is always greater than 2. So with the exception of a finite number of exceptional points $(x \in\{1,2,5,8\})$, all relevant rotors on the teeth are pointing inwards $(\downarrow)$ and the vertex $z$ receives $\left\lceil\frac{1}{2} u^{\prime}(x, 2)\right\rceil$ particles from its upper neighbour. For $x \geq 3$, the number $u^{\prime}(x, 0)$ is divisible by 4 , so all neighbours of $(x, 0)$ receive exactly the same amount of particles. Hence

$$
\begin{aligned}
\sigma_{m}(x, 1)= & \frac{1}{4} u^{\prime}(x, 0)+\frac{1}{2}\left(u^{\prime}(x, 2)+1\right)-u^{\prime}(x, 1)=\frac{1}{4}[2 f(h(x))+2 e(r(x))-2] \\
& +\frac{1}{2}[f(h(x)-2)+e(r(x)-2)+1]-f(h(x)-1)-e(r(x)-1) \\
= & \frac{1}{4}[2 h(x)(h(x)+1)+2(2 r(x)+1)-2] \\
& +\frac{1}{2}[(h(x)-2)(h(x)-1)+2(r(x)-2)+2] \\
& -(h(x)-1) h(x)-2(r(x)-1)-1=1 .
\end{aligned}
$$

if $z$ is non-exceptional. At the exceptional points $z=(x, 1)$, for $x \in\{1,2,5,8\}$, the correctness of the function $u^{\prime}$ can be verified by direct computation.

Case 3. $x \neq m$ and $y=0$ : On the $x$-axis, the points $z=(x, 0)$ for $x \in\{2,5\}$ are again exceptional and need to be checked separately. The case $x=0$ does not have to be checked at all, and $x=1$ has already been checked at the start of the proof.

For $x \notin\{2,5\}$, the vertex $z=(x, 0)$ receives particles from $(x-1,0),(x+1,0),(x, 1)$, $(x,-1)$. Here $u^{\prime}(x-1,0)$ and $u^{\prime}(x+1,0)$ are again both divisible by 4 . By symmetry $u^{\prime}(x, 1)=u^{\prime}(x,-1)$, and the number of inward pointing arrows $r(x) \geq 1$ in this case, hence 
$z$ receives $u^{\prime}(x, 1)+1$ particles from its upper and lower neighbours combined. Thus

$$
\begin{aligned}
\sigma_{m}(x, 0)= & \frac{1}{4} u^{\prime}(x-1,0)+\frac{1}{4} u^{\prime}(x+1,0)+u^{\prime}(x, 1)+1-u^{\prime}(x, 0) \\
= & \frac{1}{4}[2 f(h(x-1))+2 e(r(x-1))-2]+\frac{1}{4}[2 f(h(x+1))+2 e(r(x+1))-2] \\
& +f(h(x)-1)+e(r(x)-1))+1-[2 f(h(x))+2 e(r(x))-2] .
\end{aligned}
$$

Using that $f(x)=x(x+1)$ and $e(x)=2 x+1$ we get

$$
\begin{aligned}
\sigma_{m}(x, 0)= & \frac{1}{2}\left[h^{2}(x-1)+h^{2}(x+1)-2 h^{2}(x)\right]+\frac{1}{2}[h(x-1)+h(x+1)-6 h(x)] \\
& +[r(x-1)+r(x+1)-2 r(x)] .
\end{aligned}
$$

In order to check $\sigma_{m}(x, 0)=1$, we have to substitute in equation (12) the function $h(x)=\left\lfloor\frac{(x+1)^{2}}{3}\right\rfloor$ and the corresponding branch of the function $r(x)$ given in equation (9), depending on the congruence class mod 3 of $x$. We have to check all three cases separately. In all cases $\sigma_{m}(x, 0)=1$ holds.

Case 4. Midpoint $z=(m, 0)$ : Everything until now was independent of the number of particles $\left|B_{m}\right|$. Since $u_{m}$ is created from $u^{\prime}$ by translation and reflection, the vertex $z=(m, 0)$ after translation corresponds to the origin of the cluster. At the beginning of the process, $\left|B_{m}\right|$ particles are present at $z$, so $\sigma_{0}(z)=\left|B_{m}\right|$. We assume that $m$ is big enough, so that none of the neighbours of $z$ is an exceptional point.

By symmetry, $z=(m, 0)$ receives $\frac{1}{2} u^{\prime}(m-1,0)$ particles from its neighbours on the $x$ axis, and $u^{\prime}(m, 1)+1$ particles from its neighbours on the teeth. Hence

$$
\begin{aligned}
\sigma_{m}(m, 0)= & \sigma_{0}(m, 0)+\frac{1}{2} u^{\prime}(m-1,0)+u^{\prime}(m, 1)+1-u^{\prime}(m, 0) \\
= & \left|B_{m}\right|+\frac{1}{2}[2 f(h(m-1))+2 e(r(m-1))-2]+f(h(m)-1) \\
& +e(r(m)-1)-2 f(h(m))-2 e(r(m))+3 .
\end{aligned}
$$

Here one has to check again each congruence class mod 3 separately. Substituting the formulas for $\left|B_{m}\right|$ obtained in (4), into the previous equation, gives the desired result $\sigma_{m}(z)=1$.

Finally, we need to check that the final rotor configuration $\rho_{m}$ is acyclic. We work again with shifted coordinates. It is clear from the previous section that $\rho_{m}$ restricted to each "tooth" is acyclic. Hence it suffices to check that no cycles are created by rotors on the $x$-axis. If $z=(x, 0)$, the odometer $u_{m}(z)$ is divisible by 4 , except when $x=2$. So the rotors at these vertices point in the same direction as in the initial configuration $\rho_{0}$. The odometer at the exceptional point $w=(2,0)$ is $u^{\prime}(w)=23 \equiv 3(\bmod 4)$ independent of $m$. Hence, this rotor points in the direction of one "tooth". If the rotor at position $(2,1)$ points towards the $x$-axis, it creates a directed cycle. By $(9)$, we have $r(2)=0$, which means that all arrows on this "tooth" are pointing outwards. Hence the rotor at $w$ does not close a cycle. See Figure 3 for a visualisation of the rotor configurations under consideration.

Therefore all properties of Theorem 2.3 are satisfied and this proves the statement. 
Proof of Theorem 1.1. In the case $m \leq 2$, the statement of the Theorem follows by direct calculation of the respective aggregation clusters, see Figure 2 . For $m \geq 3$ it follows from the previous Lemma.

\section{Harmonic Measure}

In this section, as a direct application of rotor-router walks, we compute the harmonic measure of the generic set $B_{m} \subset \mathcal{C}_{2}$ defined in (1). The harmonic measure of $B_{m}$ is the hitting distribution of the set $\partial B_{m}$ for a simple random walk on $\mathcal{C}_{2}$ starting at the origin $o$.

We shall first describe the method for finite subsets $B$ of general graphs $G$, and then we apply it to the case of the comb $\mathcal{C}_{2}$ and subsets $B_{m}$ of the type defined in (1). In Theorem 1.2 , we identify the shape for which the harmonic measure is uniform. We point out that this shape does not coincide with the rotor-router aggregation cluster from Theorem 1.1. We will also describe the asymptotics of the harmonic measure for the rotor-router aggregation clusters.

In order to estimate the harmonic measure, we shall use an idea of HoLROYD AND PROPP [HP10], which they used to show a variety of inequalities concerning rotor-router walks and random walks. The method assigns a weight to the particle and rotor configuration of a rotor-router process, which is invariant under routing of particles in the system.

\subsection{Rotor Weights}

Let $G$ be a locally finite and connected graph. Start with a particle configuration $\sigma_{0}$ : $G \rightarrow \mathbb{Z}$ and a rotor configuration $\rho_{0}: G \rightarrow G$ such that $\rho_{0}(x)=x_{0}$ for all $x \in G$, that is, all initial rotors point to the first neighbour in the rotor sequence $c(x)$. We further assume that $\sigma_{0}$ has finite support, i.e., there are only finitely many particles in the system, so that we don't need to deal with questions of convergence. We will route particles in the system, and this gives rise to a sequence $\left(\rho_{t}, \sigma_{t}\right)_{t \geq 0}$ of particle and rotor configurations at every time $t$. To each of the possible states $\left(\rho_{t}, \sigma_{t}\right)$ of the system, we will assign a weight.

Fix a function $\psi: G \rightarrow \mathbb{R}$. We define the particle weights at time $t$ to be

$$
\mathbf{W}_{\mathbf{P}}(t)=\sum_{x \in G} \sigma_{t}(x) \psi(x)
$$

Further define the rotor weights of vertices $x \in G$ as

$$
w(x, k)= \begin{cases}0, & \text { for } k=0 \\ w(x, k-1)+\psi(x)-\psi\left(x_{k \bmod d(x)}\right), & \text { for } k>0\end{cases}
$$

where $x_{i}$ is the $i$-th neighbour of $x$ in the rotor sequence $c(x)$. Notice that, for $k \geq d(x)$,

$$
w(x, k)=w(x, k-d(x))-d(x) \triangle \psi(x) .
$$


Here $\triangle \psi(x)$ represents the Laplace operator which is defined as

$$
\triangle \psi(x)=\frac{1}{d(x)} \sum_{y \in G: y \sim x}(\psi(y)-\psi(x)) .
$$

The total rotor weights at time $t$ are given by

$$
\mathbf{W}_{\mathbf{R}}(t)=\sum_{x \in G} w\left(x, u_{t}(x)\right)
$$

where $u_{t}(x)$ is the odometer function of this process, that is, the number of particles sent out by the vertex $x$ in the first $t$ steps. Note that $\rho_{0}$ is chosen in such a way that for all $t \geq 0$ and $x \in G$, if $i \equiv u_{t}(x) \bmod d(x)$, then $x_{i}=\rho_{t}(x)$.

It is easy to check that the sum of particle and rotor weights are invariant under routing of particles, i.e., for all times $t, t^{\prime} \geq 0$

$$
\mathbf{W}_{\mathbf{P}}(t)+\mathbf{W}_{\mathbf{R}}(t)=\mathbf{W}_{\mathbf{P}}\left(t^{\prime}\right)+\mathbf{W}_{\mathbf{R}}\left(t^{\prime}\right) .
$$

\subsection{Harmonic Measure for finite subsets of graphs}

As before, let $G$ be a locally finite, connected graph, and let $B$ be some finite subset of G. Write

$$
\partial B=\{x \in B: \exists y \notin B \text { with } x \sim y\}
$$

for the inner boundary of $B$, and $B^{\circ}=B \backslash \partial B$. The vertices of $\partial B$ will represent the sink $S$.

Similarly to Definition 2.1 of Section 2, we define the particle addition operator $E_{x}$, for each vertex $x \in B^{\circ}$, as follows: for a rotor configuration $\rho$, let

$$
E_{x}(\rho)=\rho^{\prime}
$$

where $\rho^{\prime}$ is the rotor configuration obtained from $\rho$ by adding a new particle at vertex $x$, and letting it perform a rotor-router walk until the particle reaches a vertex in $\partial B$ for the first time. By the abelian property of rotor-router walks the operators $E_{x}$ commute, and they can be used to define an abelian group, see [HLM $\left.{ }^{+} 08\right]$ for details and $\mathrm{HLM}^{+} 08$, Lemma 3.10] for the proof of the following statement.

Lemma 4.1. The particle addition operator $E_{x}$ is a permutation on the set of acyclic rotor configurations on $B^{\circ}$.

The rotor-router group of $B^{\circ}$ is defined as the subgroup of permutations of oriented spanning trees rooted at the sink (that is, acyclic rotor configurations) generated by $\left\{E_{x}: x \in B^{\circ}\right\}$. For every finite graph $B^{\circ}$ the rotor-router group is a finite abelian group, which is isomorphic to the abelian sandpile group. See once again [HLM $\left.{ }^{+} 08\right]$ for details.

Consider the simple random walk $\left(X_{t}\right)_{t \geq 0}$ on $G$, i.e., a Markov chain with state space $G$, and transition probabilities given by

$$
p(x, y)=\frac{1}{d(x)}, \quad \text { for all } x, y \in G, \text { with } x \sim y .
$$

THE ELECTRONiC Journal of COMBinatorics 18 (2011), \#P224 
Then $X_{t}$ is a $G$-valued random variable, and represents the random position of the random walker at the discrete time $t$. For given $x \in G$, we write $\mathbb{P}_{x}$ for the law of a random walk starting at $x$. Consider the stopping time

$$
T=\inf \left\{t \geq 0: X_{t} \in \partial B\right\}
$$

For $z \in \partial B$, let

$$
\nu_{x}(z)=\mathbb{P}_{x}\left[X_{T}=z\right],
$$

be the harmonic measure at $z$ with starting point $x$, that is, the probability that a random walk starting at $x$ hits $\partial B$ for the first time in $z$.

Take the harmonic measure itself as the weight function. More explicitly, fix a vertex $z \in \partial B$, and define the weight function $\psi(x)$ as

$$
\psi(x)=\psi_{z}(x)=\nu_{x}(z) .
$$

Let us define the following process. Start with $n$ particles at the origin $o$, and an arbitrary acyclic rotor configuration $\rho_{0}$. Let the particles perform rotor-router walks until they reach a vertex in $\partial B$ for the first time, where they stop. Denote by $t^{\star}=t^{\star}(n)$ the number of steps this process takes to complete, and for each $w \in \partial B$, write $e(w)$ for the number of particles that stopped in $w$ at the end of this procedure. We denote by $\hat{u}$ the normalized rotor-router odometer function of this process, that is, for all $x \in B$,

$$
\hat{u}(x)=\frac{\text { number of particles sent out by } x}{d(x)} .
$$

Using the invariance of the sum of rotor and particle weights under rotor-router walks, as in 16 , we get

$$
n \psi(o)=\sum_{w \in \partial B} e(w) \psi(w)+\mathbf{W}_{\mathbf{R}}\left(t^{\star}\right),
$$

since $\mathbf{W}_{\mathbf{R}}(0)=0, \mathbf{W}_{\mathbf{P}}(0)=n \psi(0)$ and $\mathbf{W}_{\mathbf{P}}\left(t^{\star}\right)=\sum_{w \in \partial B} e(w) \psi(w)$. Equation (17) reduces to

$$
n \psi(o)=e(z)+\mathbf{W}_{\mathbf{R}}\left(t^{\star}\right),
$$

because $\psi(w)=\nu_{w}(z)=\delta_{w}(z)$, if $w \in \partial B$.

The initial rotor configuration $\rho_{0}$ is chosen to be acyclic. Therefore, there exists a number $n$ such that, after all $n$ particles performed their rotor-router walks, all rotors in $B^{\circ}$ made only full turns, i.e., $\rho_{0}=\rho_{t}^{\star}$. This claim follows from Lemma 4.1. Hence, $n$ is a multiple of the order of $E_{o}$ in the rotor-router group. Since $\psi$ is a harmonic function on $B^{\circ}$, using a $n$ with the above property gives $\mathbf{W}_{\mathbf{R}}\left(t^{\star}\right)=0$, which together with (18) leads to

$$
n \cdot \nu_{o}(z)=e(z) .
$$

Thus, the harmonic measure $\nu_{o}$ of $B$ is proportional to the number of particles which stopped at $\partial B$. While a number $n$ with the right property is difficult to calculate, we can still use equation (19) in order to derive asymptotics of the harmonic measure of subsets $B_{m}$ of the comb $\mathcal{C}_{2}$, and in some cases even to calculate it explicitly. 


\subsection{Subsets of the Comb}

Let us consider subsets $B_{m}$ of $\mathcal{C}_{2}$, of the type defined in (1), with generic positive function $h: \mathbb{N}_{0} \rightarrow \mathbb{N}_{0}$. Recall here the definition of $B_{m}$.

$$
B_{m}=\left\{(x, y) \in \mathcal{C}_{2}:|x| \leq m,|y| \leq h(m-|x|)\right\} \quad \text { for } m \in \mathbb{N} .
$$

By construction, all rotors make only full turns if we perform the rotor-router process from Section 4.2, for the set $B_{m}$. This implies that the corresponding normalized odometer function $\hat{u}$ is harmonic outside the origin and its Laplacian is given by

$$
\triangle \hat{u}(w)= \begin{cases}0, & w \in B_{m} \backslash\left(\partial B_{m} \cup\{o\}\right) \\ -n, & w=o,\end{cases}
$$

and $\hat{u}(w)=0$, for $w \in \partial B_{m}$. Write $\nu_{m, o}(w)$ for the harmonic measure of $B_{m}$ and $e_{m}(w)$ for the number of rotor-router particles stopped in $w \in \partial B_{m}$. By symmetry of the set $B_{m}$, it is clear that also $e_{m}(w)$ and $\nu_{m, o}(w)$ are symmetric. More precisely, if $w=(x, y)$ and $w^{\prime}=(|x|,|y|)$ then

$$
e_{m}(w)=e_{m}\left(w^{\prime}\right) \quad \text { and } \quad \nu_{m, o}(w)=\nu_{m, o}\left(w^{\prime}\right) .
$$

Hence it is enough to work in one quadrant. We will choose the second quadrant (i.e. $x \leq 0$ and $y \geq 0$ ), and for simplicity of notation shift the set $B_{m}$ by $m$ in the direction of the positive $x$ axis, such that the leftmost point of $B_{m}$ has coordinate $(0,0)$ and its center $o$ has coordinate $(m, 0)$. So, the set under consideration is now

$$
B_{m}^{\square}=\left\{(x, y) \in \mathcal{C}_{2}: 0 \leq x \leq m, 0 \leq y \leq h(x)\right\}
$$

We will also use $\hat{u}$ for the normalized odometer function and $e_{m}$ for the number of particles which hit boundary points in the shifted coordinate system. Additionally, since $e_{m}$ is defined only on $\partial B_{m}$ we write $e_{m}(x)=e_{m}(x, h(x))$, for $0 \leq x \leq m$.

Solving the Dirichlet problem (20) on the "teeth" of the comb, gives for $(x, y) \in B_{m}^{\square}$,

$$
\hat{u}(x, y)=e_{m}(x) \cdot(h(x)-y)
$$

On the $x$ axis, for $(x, 0) \neq o$, the harmonicity gives

$$
\hat{u}(x+1,0)+\hat{u}(x-1,0)+2 \hat{u}(x, 1)=4 \hat{u}(x, 0),
$$

which together with (21) leads to the following recursion for $e_{m}(x)$ and $0<x<m$ :

$$
e_{m}(x+1) h(x+1)+e_{m}(x-1) h(x-1)-2 e_{m}(x)(h(x)+1)=0 .
$$

We are now ready prove Theorem 1.2 , which characterizes sets of uniform harmonic measure. 
Proof of Theorem 1.2. Let $B_{m}$ be defined as in (1), with $h(x)=x^{2}$. From (22) we get the recursion

$$
e_{m}(x+1)(x+1)^{2}+e_{m}(x-1)(x-1)^{2}-2 e_{m}(x)\left(x^{2}+1\right)=0, \quad \text { for } 0<x<m .
$$

Since $h(1)=1$, the vertex $z=(1,0)$ in the shifted coordinate system has three neighbours on the boundary $\partial B_{m}$. By construction, the rotor at $(1,0)$ makes a number of full turns, hence all of these three neighbours receive the same amount of particles from $z$. Therefore $e_{m}(0)=e_{m}(1)$. By induction, it is easy to see that the sequence $e_{m}(x)$ is constant. Assuming $e_{m}(x-1)=e_{m}(x)$, the recursion (23) reduces to

$$
e_{m}(x+1)(x+1)^{2}-e_{m}(x)(x+1)^{2}=0 .
$$

which implies that $e_{m}(x+1)=e_{m}(x)$. Because $e_{m}(x)$ is by construction proportional to the harmonic measure $\nu_{m, o}$, we get the claim.

In general we can compute the harmonic measure for all sets $B_{m}$, where (24) can be solved exclicitly.

\subsection{Harmonic Measure of the Rotor-Router Cluster}

This section is dedicated to the proof of Theorem 1.3. Recall that the rotor-router cluster obtained in Theorem 1.1 is a set of type $B_{m}$, as defined in (1), with $h(x)=\left\lfloor(x+1)^{2} / 3\right\rfloor$.

Like before, let $e_{m}(x)$ be the number of particles stopped at boundary points $(x, h(x)) \in$ $\partial B_{m}$ in the rotor-router process defined in Section 4.2. By linearity also the normalized sequence $e(x)=\frac{e_{m}(x)}{e_{m}(0)}$ is a solution of the recurrence 22 , and since $h(1)=1$ we have $e(0)=e(1)=1$. Hence the function $e(x)$ is independent of $m$. Rewriting (22) in this case, we get a linear recurrence with non-polynomial coefficients. While an explicit answer is not feasible, we can derive asymptotics of the special solution $e(x)$, by converting the recurrence into an equivalent system of linear differential equations. We will prove Theorem 1.3 by showing that the function $e(x)$ has linear growth, which is accomplished in the next two Lemmas.

Lemma 4.2. There exists a constant $c<\frac{1}{2}$ such that

$$
\frac{e(x)}{x} \rightarrow c \text { as } x \rightarrow \infty .
$$

Proof. Substitute $\tilde{e}(x)=\frac{e(x)}{x}$ for $x>0$, which transforms 22 into

$$
\tilde{e}(x-1)(x-1) h(x-1)+\tilde{e}(x+1)(x+1) h(x+1)-2 \tilde{e}(x) x(h(x)+1)=0 .
$$

The sequence $\tilde{e}(x)$ converges if and only if $e(x)$ grows at most linearly. Since $e(x)$ is positive by construction, it suffices to check that $\tilde{e}(x)$ is decreasing. For this, consider the auxiliary function $h^{\prime}(x)=\frac{(x+1)^{2}}{3}-\frac{1}{3}$. We have to distinguish three cases

$$
h(x)=\left\{\begin{array}{lll}
h^{\prime}(x), & x \equiv 0 & \bmod 3 \\
h^{\prime}(x), & x \equiv 1 & \bmod 3 \\
h^{\prime}(x)+\frac{1}{3}, & x \equiv 2 & \bmod 3
\end{array}\right.
$$


We prove the monotonicity of $\tilde{e}(x)$ by induction. Assuming $\tilde{e}(x)<\tilde{e}(x-1)$ for $x \equiv 0$ $\bmod 3$ we show that $\tilde{e}(x+3)<\tilde{e}(x+2)<\tilde{e}(x+1)<\tilde{e}(x)$. The induction base follows by calculating the first elements of the sequence.

Case 1. Assume $x \equiv 0 \bmod 3$ and $\tilde{e}(x)<\tilde{e}(x-1)$. Then (24) can be rewritten as

$$
\tilde{e}(x+1)(x+1) h^{\prime}(x+1)=2 \tilde{e}(x) x\left(h^{\prime}(x)+1\right)-\tilde{e}(x-1)(x-1)\left(h^{\prime}(x-1)+\frac{1}{3}\right) .
$$

Using the induction hypothesis and the definition of $h^{\prime}(x)$, we get

$$
\tilde{e}(x+1)<\tilde{f}_{0}(x) \cdot \tilde{e}(x),
$$

with $\tilde{f}_{0}(x)=\frac{x^{2}+2 x}{x^{2}+2 x+1}<1$, which implies $\tilde{e}(x+1)<\tilde{e}(x)$.

Case 2. Assume $x \equiv 1 \bmod 3$ and $\tilde{e}(x)<\tilde{f}_{0}(x-1) \cdot \tilde{e}(x-1)$. Like before, rewrite (24) as

$$
\tilde{e}(x+1)(x+1)\left(h^{\prime}(x+1)+\frac{1}{3}\right)=2 \tilde{e}(x) x\left(h^{\prime}(x)+1\right)-\tilde{e}(x-1)(x-1) h^{\prime}(x-1) .
$$

This gives, by 25

$$
\tilde{e}(x+1)<\tilde{f}_{1}(x) \cdot \tilde{e}(x),
$$

for

$$
\begin{aligned}
\tilde{f}_{1}(x) & =\frac{2 x\left(h^{\prime}(x)+1\right)-\tilde{f}_{0}(x-1)^{-1}(x-1) h^{\prime}(x-1)}{(x+1)\left(h^{\prime}(x+1)+\frac{1}{3}\right)} \\
& =\frac{x^{2}+3 x}{x^{2}+3 x+2}<1,
\end{aligned}
$$

which implies $\tilde{e}(x+1)<\tilde{e}(x)$.

Case 3. Finally, assuming $x \equiv 2 \bmod 3$ and $\tilde{e}(x)<\tilde{f}_{1}(x-1) \cdot \tilde{e}(x-1)$, we get

$$
\tilde{e}(x+1)(x+1) h^{\prime}(x+1)=2 \tilde{e}(x) x\left(h^{\prime}(x)+\frac{4}{3}\right)-\tilde{e}(x-1)(x-1) h^{\prime}(x-1) .
$$

Applying (26), we obtain

$$
\tilde{e}(x+1)<\tilde{f}_{2}(x) \cdot \tilde{e}(x),
$$

for the function $\tilde{f}_{2}(x)=\frac{x^{4}+7 x^{3}+17 x^{2}+17 x}{x^{4}+7 x^{3}+17 x^{2}+17 x+6}<1$.

This shows that $\tilde{e}(x)$ is decreasing and therefore convergent, which also means that there exists a constant $c$ such that $\frac{e(x)}{x} \rightarrow c$, as $x \rightarrow \infty$. The fact that $c<\frac{1}{2}$ follows by computing the first few values of the sequence $e(x)$, using $e(0)=e(1)=1$ as starting values in the recursion (22). By monotonicity we then get $\tilde{e}(x)<\frac{1}{2}$ for all $x \geq 20$.

The next result shows that the function $e(x)$ has at least linear growth.

Lemma 4.3. The constant c in Lemma 4.2 is strictly positive. 
Proof. To show that $c>0$, we use singularity analysis of linear differential equations. For this, we split $e(x)$ into three sequences modulo 3 , i.e., for $k \in \mathbb{N}$ write

$$
e_{i}(k)=e(3 k+i) \quad \text { for } i=0,1,2 \text {, }
$$

and rewrite 22 for each congruence class of $x \bmod 3$ in terms of $k$. This leads to a system of linear recursions which can be written in matrix form as

$$
A_{k} \cdot \vec{e}(k-1)=B_{k} \cdot \vec{e}(k),
$$

with $\vec{e}(k)=\left(e_{0}(k), e_{1}(k), e_{2}(k)\right)^{t}$, and the matrices $A_{k}$ and $B_{k}$ given as

$$
A_{k}=\left(\begin{array}{ccc}
0 & 3 k^{2}-2 k & -6 k^{2}-2 \\
0 & 0 & 3 k^{2} \\
0 & 0 & 0
\end{array}\right), \quad B_{k}=\left(\begin{array}{ccc}
-3 k^{2}-2 k & 0 & 0 \\
6 k^{2}+4 k+2 & -3 k^{2}-4 k-1 & 0 \\
3 k^{2}+2 k & -6 k^{2}-8 k-4 & 3 k^{2}+6 k+3
\end{array}\right) .
$$

The initial values are given by $\vec{e}(0)=\left(1,1, \frac{4}{3}\right)^{t}$. Denote by $E_{i}(z)=\sum_{k \geq 0} e_{i}(k) z^{k}$ the generating function of $e_{i}(k), i=0,1,2$. Using the identities

$$
\sum_{k \geq 0} k e_{i}(k) z^{k}=z \frac{\partial}{\partial z} E_{i}(z) \text { and } \sum_{k \geq 0} k^{2} e_{i}(k) z^{k}=z^{2} \frac{\partial^{2}}{\partial z^{2}} E_{i}(z)+z \frac{\partial}{\partial z} E_{i}(z)
$$

the matrix recursion $(28)$ can be transformed into the following system of linear differential equations for the generating functions $E_{i}(k)$

$$
C \cdot \vec{E}(z)=b
$$

where $\vec{E}(z)=\left(E_{0}(z), E_{1}(z), E_{2}(z)\right)^{t}$, and $C$ is a matrix of linear differential operators given as

$$
C=\left(\begin{array}{ccc}
5 \frac{\partial}{\partial z}+3 z \frac{\partial^{2}}{\partial z^{2}} & 1+7 z \frac{\partial}{\partial z}+3 z^{2} \frac{\partial^{2}}{\partial z^{2}} & -8-18 z \frac{\partial}{\partial z}-6 z^{2} \frac{\partial^{2}}{\partial z^{2}} \\
-2-10 z \frac{\partial}{\partial z}-6 z^{2} \frac{\partial^{2}}{\partial z^{2}} & 1+7 z \frac{\partial}{\partial z}+3 z^{2} \frac{\partial^{2}}{\partial z^{2}} & 3 z+9 z^{2} \frac{\partial}{\partial z}+3 z^{3} \frac{\partial^{2}}{\partial z^{2}} \\
5 z \frac{\partial}{\partial z}+3 z^{2} \frac{\partial^{2}}{\partial z^{2}} & -4-14 z \frac{\partial}{\partial z}-6 z^{2} \frac{\partial^{2}}{\partial z^{2}} & 3+9 z \frac{\partial}{\partial z}+3 z^{2} \frac{\partial^{2}}{\partial z^{2}}
\end{array}\right), \quad b=\left(\begin{array}{c}
0 \\
e_{1}(0)-2 e_{0}(0) \\
0
\end{array}\right) .
$$

To solve (29) asymptotically, we consider $C$ as a matrix with entries in the Weyl algebra, that is, the noncommutative ring of linear differential operators with polynomial coefficients, see [Lam91]. We can perform a division-free Gauss elimination over this ring to transform $C$ into row echelon form, which gives a single differential equation only involving $E_{2}(z)$. The actual computations were performed using the computer algebra system FriCAS The result is a differential equation of order 7 for $E_{2}(z)$ :

\footnotetext{
${ }^{1}$ http://fricas.sourceforge.net
} 


$$
\begin{array}{r}
\frac{81}{8}(z+2)(z-1)^{5} z^{6} \frac{\partial^{7}}{\partial z^{7}} \\
+\frac{1269}{4}(z-1)^{4} z^{5}\left(z^{2}+z-\frac{76}{47}\right) \frac{\partial^{6}}{\partial z^{6}} \\
+\frac{27531}{8}(z-1)^{3} z^{4}\left(z^{3}-\frac{24}{437} z^{2}-\frac{7149}{3059} z+\frac{3826}{3059}\right) \frac{\partial^{5}}{\partial z^{5}} \\
+\frac{127725}{8} \cdot(z-1)^{2} \cdot z^{3} \cdot\left(z^{4}-\frac{50039}{42575} z^{3}-\frac{82401}{42575} z^{2}+\frac{132307}{42575} z-\frac{38554}{42575}\right) \frac{\partial^{4}}{\partial z^{4}} \\
+31785(z-1) z^{2}\left(z^{5}-\frac{100697}{42380} z^{4}-\frac{1164}{10595} z^{3}+\frac{36215}{8476} z^{2}-\frac{5651}{1630} z+\frac{6234}{10595}\right) \frac{\partial^{3}}{\partial z^{3}} \\
+23970 z \cdot\left(z^{6}-\frac{117579}{31960} z^{5}+\frac{114057}{31960} z^{4}+\frac{15053}{6392} z^{3}-\frac{208329}{31960} z^{2}+\frac{59229}{15980} z-\frac{1243}{3995}\right) \frac{\partial^{2}}{\partial z^{2}} \\
+4935\left(z^{6}-\frac{1354}{329} z^{5}+\frac{1843}{329} z^{4}-\frac{4479}{3290} z^{3}-\frac{12209}{3290} z^{2}+\frac{1466}{329} z-\frac{32}{329}\right) \frac{\partial}{\partial z} \\
+105\left(z^{5}-\frac{494}{105} z^{4}+\frac{881}{105} z^{3}-\frac{201}{70} z^{2}+\frac{4411}{210} z+\frac{1006}{105}\right)=0
\end{array}
$$

Using singularity analysis for linear differential equations, we can derive asympotics of $e_{2}(k)$. See Flajolet And SEDGewick [FS09, Theorem VII.10] for details. The coefficient of the highest order term $\frac{\partial^{7}}{\partial z^{7}}$ is given by

$$
\frac{81}{8}(z+2)(z-1)^{5} z^{6}
$$

hence the dominant non-zero singularity $\xi$ is equal to 1 . Since all coefficients in (30) are given in factorized form, it is immediate that $\xi$ is a regular singularity. Calculating the indicial polynomial for the singularity $\xi$ gives

$$
I_{\xi}(\theta)=\theta^{7}-17 \theta^{6}+99 \theta^{5}-187 \theta^{4}-220 \theta^{3}+1044 \theta^{2}-720 \theta .
$$

For the definition of a regular singularity and the indicial polynomial, see once again Flajolet And SEdGewiCK [FS09, Chapter VII.9]. The roots of $I_{\xi}(\theta)$ are $-2,0,1,3,4,5$ and 6 . Since they differ by integers, the asymptotics of $e_{2}(k)$ is given by

$$
e_{2}(k) \sim c \cdot \xi^{-k} k^{\beta} \log ^{l} k
$$

where $l$ is an integer and $\beta$ is the biggest solution of the equation $I(-\beta-1)=0$, see [FS09, page 521, equation 118]. The $\sim \operatorname{sign}$ means "approximately equal" (in the precise sense that the ratio of both terms tends to 1 as $k$ gets large).

In our case $\beta=1$, and we have

$$
e_{2}(k) \sim c \cdot k \log ^{l} k
$$

and this proves the desired.

While it is not known how to calculate the constant $l$ in 31 in the general case, from Lemma 4.2 we already know that $e_{2}(k)$ grows at most linearly, hence $l=0$. Therefore, Lemma 4.2 and Lemma 4.3 together imply Theorem 1.3 . 
Acknowledgements: We are grateful to Franz Lehner for interesting discussions and for helping with the computer computations in the proof of Lemma 4.3, and also to the anonymous referee whose comments led to an essential improvement of the paper.

The research of Wilfried Huss and Ecaterina Sava was partially supported by the Austrian Science Fund (FWF): W1230-N13.

\section{References}

[Ber06] D. Bertacchi, Asymptotic behaviour of the simple random walk on the 2dimensional comb, Electron. J. Probab. 11, no. 45, 1184-1203 (2006).

[BTW88] P. Bak, C. Tang and K. Wiesenfeld, Self-organized criticality, Phys. Rev. A 38(1), 364-374 (1988).

[CS06] J. N. Cooper and J. Spencer, Simulating a Random Walk with Constant Error, Combinatorics, Probability and Computing 15, 815-822 (2006).

[DF06] B. Doerr and T. Friedrich, Deterministic Random Walks on the TwoDimensional Grid, in ISAAC, edited by T. Asano, volume 4288 of Lecture Notes in Computer Science, pages 474-483, Springer, 2006.

[FL11] T. Friedrich and L. Levine, Fast Simulation of Large-Scale Growth Models, in APPROX-RANDOM, edited by L. A. Goldberg, K. Jansen, R. Ravi and J. D. P. Rolim, volume 6845 of Lecture Notes in Computer Science, pages 555-566, Springer, 2011.

[FS09] P. Flajolet and R. Sedgewick, Analytic Combinatorics, Cambridge Univ. Press, Cambridge, 2009.

$\left[\mathrm{HLM}^{+} 08\right]$ A. E. Holroyd, L. Levine, K. Mészáros, Y. Peres, J. Propp and D. B. Wilson, Chip-firing and rotor-routing on directed graphs, in In and out of equilibrium. 2, volume 60 of Progr. Probab., pages 331-364, Birkhäuser, Basel, 2008.

[HP10] A. E. Holroyd and J. Propp, Rotor Walks and Markov Chains, in Algorithmic Probability and Combinatorics, edited by Robert S. Maier, M. Marni, M. E. Lladser and A. Rechnitzer, volume 520 of Contemporary Mathematics, pages 105-126, Amer. Math. Soc., 2010.

[HS11] W. Huss and E. Sava, Internal Aggregation Models on the Comb Lattice, (2011), arXiv:1106.4468.

[KL10] W. Kager and L. Levine, Rotor-Router Aggregation on the layered square lattice, The Electronic Journal of Combinatorics 17(1), R152 (2010).

[KP04] M. Krishnapur and Y. Peres, Recurrent Graphs where Two Independent Random Walks Collide Finitely Often, Electronic Communications in Probability 9, 72-81 (2004).

[Lam91] T. Y. Lam, A first course in noncommutative rings, volume 131 of Graduate texts in mathematics, Springer, New York, 1991. 
[LL09] I. Landau and L. Levine, The rotor-router model on regular trees, J. Combin. Theory Ser. A 116(2), 421-433 (2009).

[LP09] L. Levine and Y. Peres, Strong Spherical Asymptotics for Rotor-Router Aggregation and the Divisible Sandpile, Potential Analysis 30(1), 1-27 (2009).

[PDDK96] V. B. Priezzhev, D. Dhar, A. Dhar and S. Krishnamurthy, Eulerian Walkers as a Model of Self-Organized Criticality, Phys. Rev. Lett. 77(25), 5079-5082 (1996).

[PPS98] A. M. Povolotsky, V. B. Priezzhev and R. R. Shcherbakov, Dynamics of Eulerian walkers, Phys. Rev. E 58(5), 5449-5454 (1998). 